\title{
Efficacy and safety of switching to latanoprost $0.005 \%$-timolol maleate $0.5 \%$ fixed-combination eyedrops from an unfixed combination for 36 months
}

Kenji Inoue'

Ryoko Okayama'

Risako Higa'

Goji Tomita ${ }^{2}$

'Inouye Eye Hospital, Tokyo, ${ }^{2}$ Second Department of Ophthalmology, Toho University School of Medicine, Tokyo, Japan
Correspondence: Kenji Inoue Inouye Eye Hospital, 4-3 Kanda-Surugadai, Chiyoda-ku, Tokyo I0I-0062, Japan

Tel +8I 33295 09। I

$\mathrm{Fax}+81332950917$

Email inoue-k@inouye-eye.or.jp
This article was published in the following Dove Press journal:

Clinical Ophthalmology

3 July 2014

Number of times this article has been viewed

Purpose: We prospectively investigated the intraocular pressure (IOP)-reducing effect, the visual field-maintenance effect, and the adverse reactions caused by administration of latanoprost/ timolol maleate fixed-combination eyedrops for 3 years.

Subjects and methods: The subjects were 162 patients (162 eyes) with glaucoma or ocular hypotension who were using latanoprost and timolol maleate eyedrops concomitantly. The latanoprost and timolol maleate eyedrop regimen was discontinued without any washout period and subsequently changed to a latanoprost-timolol maleate fixed-combination eyedrop regimen. IOP values before the change and at $6,12,18,24,30$, and 36 months after the change were measured and compared. The Humphrey visual field test was conducted before the change and at 12, 24, and 36 months after the change, and mean-deviation values were compared. Adverse reactions were assessed at every checkup.

Results: The IOPs before the change and at 6-36 months after the change were $15.2 \pm 3.3 \mathrm{mmHg}$ and $14.3-15.2 \mathrm{mmHg}$, respectively, and a significant decrease was observed at 36 months after the change $(P<0.01)$. Mean-deviation values before the change and at $12-36$ months after the change were $-9.11 \pm 6.94 \mathrm{~dB}$ and -10.08 to $-9.22 \mathrm{~dB}$, respectively, and these values were not significantly different $(P=0.2148)$. Within the 36-month period, the eyedrops were discontinued because of insufficient IOP decrease in 33 cases (20.4\%) and adverse reactions in eleven cases $(6.8 \%)$.

Conclusion: IOP and the visual field were maintained for 3 years when a latanoprost and timolol maleate eyedrop regimen was changed to a latanoprost-timolol maleate fixed-combination eyedrop regimen. However, administration of the latanoprost-timolol maleate fixed-combination eyedrops was discontinued in approximately $27 \%$ of cases because of insufficient IOP decrease and adverse reactions.

Keywords: latanoprost $0.005 \%$-timolol maleate $0.5 \%$ fixed combination, intraocular pressure, safety, visual field

\section{Introduction}

Glaucoma is a chronic and progressive disease, and patients are required to receive treatment throughout their lifetime because complete recovery is not possible. The first choice in glaucoma treatment is eyedrops. Treatment with eyedrops begins as monotherapy, but other drugs are added and multidrug therapy is required in cases in which the intraocular pressure (IOP) remains high. Decreased adherence is a problem in multidrug therapy, ${ }^{1}$ and fixed-combination eyedrops were developed for the purpose of improving adherence. The first eyedrops that were introduced on the market in 
Japan were a fixed combination of latanoprost and timolol maleate eyedrops (Xalacom ${ }^{\circledR}$; New York, NY, USA) in 2010. Numerous studies ${ }^{2-7}$ have reported a decrease in IOP when a latanoprost and timolol maleate eye-drop regimen was changed to a latanoprost-timolol maleate fixed-combination eyedrop regimen. However, few reports ${ }^{2,3}$ have assessed the effects of an administration period longer than 6 months after the change. Moreover, although a long-term follow-up period is needed when investigating the visual field-maintenance effect, only our previous report ${ }^{2}$ assessed an administration period of 1 year.

In the present study, the subjects were primary open-angle glaucoma or ocular hypertension patients who were using latanoprost and timolol maleate eyedrops concomitantly and were subsequently asked to use latanoprost-timolol maleate fixed-combination eyedrops. The assessment period of 1 year in our previous study ${ }^{2}$ was extended to 3 years, and the IOP-decreasing efficacy, visual field-maintenance effect, and safety of the fixed-combination eyedrop regimen were prospectively investigated.

\section{Subjects and methods}

The subjects in this study were the same as those described in our previous report. ${ }^{2}$ We prospectively investigated 162 cases (162 eyes: 88 men [88 eyes], 74 women [74 eyes]) that presented to Inouye Eye Hospital from April to October 2010. The mean age of the subjects was $64.3 \pm 11.4$ years (average \pm mean deviation [MD]; range 32-87 years). The disease type was normal-tension glaucoma in 65 cases, primary open-angle glaucoma in 93 cases, and ocular hypertension in four cases. The number of previously used glaucoma eyedrop medications was two in 91 cases, three in 58 cases, and four in 13 cases. The patients had used timolol maleate eyedrops in the following formulations: aqueous timolol eyedrops in 38 cases, ionic-reply gel timolol eyedrops in 105 cases, and heat-reply gel timolol eye drops in 19 cases. The MD value according to the Humphrey field analysis program 30-2 Swedish Interactive Threshold Algorithms (SITA) standard was $-9.11 \pm 6.94 \mathrm{~dB}$ (range -32.47 to $1.03 \mathrm{~dB}$ ). The IOP before changing to the latanoprost-timolol maleate fixed-combination eyedrop regimen was $15.2 \pm 3.3 \mathrm{mmHg}$ (8-32 mmHg). If both eyes met the inclusion criteria, the eye with the higher IOP was selected. If both eyes had the same IOP, the right eye was selected. If one eye met the requirements, the corresponding eye was subjected to analysis.

The latanoprost and timolol maleate eyedrops that the patients had been using were discontinued without any washout period and changed to latanoprost $0.005 \%$-timolol maleate $0.5 \%$ fixed-combination eyedrops (administration once a day at night). All other eyedrops except latanoprost and timolol maleate eyedrops were continued. The IOP was measured before administration and at every 1-3 months after administration; measurements were performed with a Goldmann applanation tonometer by the same examiner at approximately the same time of day. The IOP before administration was compared with that at 6, 12, 18, 24, and 30 months after administration by using analysis of variance and the Bonferroni/Dunn test. The Humphrey field analysis program 30-2 SITA standard was conducted before and at 6 , $12,18,24,30$, and 36 months after the change, and the MDs were compared before and at 12, 24, and 36 months after the change by using Student's paired $t$-test. Adverse reactions were assessed at every checkup, and the reason was examined in the discontinued cases. The continuous activity rate of latanoprost-timolol maleate fixed-combination eyedrops was investigated using the Kaplan-Meier method. Moreover, the reason for discontinuation and the period of discontinuation were investigated. The significance level was set at $P<0.05$. This study was approved by the ethics committee of Inouye Eye Hospital. The purpose and content of the study were explained to the patients, and informed consent was obtained from the patients before conducting the study.

\section{Results}

The IOP was $15.2 \pm 3.0 \mathrm{mmHg}$ after 6 months, $14.9 \pm 3.4 \mathrm{mmHg}$ after 12 months, $15.0 \pm 3.1 \mathrm{mmHg}$ after 18 months, $14.8 \pm 3.4 \mathrm{mmHg}$ after 24 months, $14.4 \pm 2.9 \mathrm{mmHg}$ after 30 months, and $14.3 \pm 3.0 \mathrm{mmHg}$ after 36 months. When compared to the IOP before administration $(15.2 \pm 3.3 \mathrm{mmHg}$ ), the decrease in IOP was significant only at 36 months after administration $(P<0.01)$. When the individual IOP values obtained before the change were compared with those after 36 months, the IOP decreased more than $2 \mathrm{mmHg}$ in 13 cases $(13.0 \%)$, was within $2 \mathrm{mmHg}$ in 77 cases $(77.0 \%)$, and increased more than $2 \mathrm{mmHg}$ in ten cases $(10.0 \%)$ (Figure 1).

MD values were $-9.11 \pm 6.94 \mathrm{~dB}$ before the change, $-10.08 \pm 7.24 \mathrm{~dB}$ at 12 months after the change, $-9.96 \pm 7.35 \mathrm{~dB}$ at 24 months after the change, and $-9.22 \pm 7.35 \mathrm{~dB}$ at 36 months after the change. No significant differences were found between these values $(P=0.2148)$.

In 62 cases $(38.3 \%)$, the eyedrops were discontinued within 36 months after the change (Table 1). The reason for discontinuation was an insufficient decrease in IOP in 33 cases $(20.4 \%)$, adverse reactions in eleven cases $(6.8 \%)$, discontinuation of patient visits to the hospital in 15 cases 


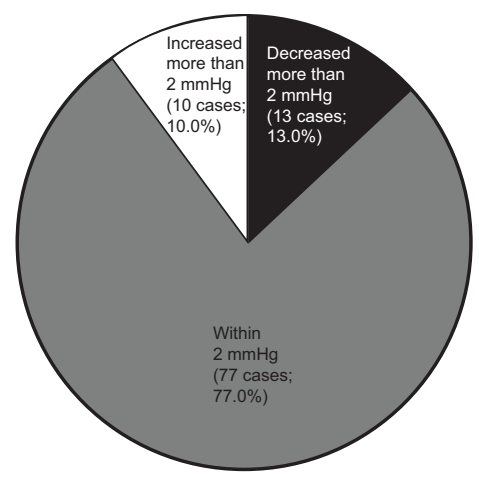

Figure I Decrease in intraocular pressure at 36 months after the change to latanoprost-timolol maleate fixed-combination eyedrops.

$(9.3 \%)$, and cataract surgery in three cases $(4.8 \%)$. The adverse reactions were ophthalmalgia in three cases, itchiness in two cases, a sensation of stimulation in two cases, photophobia in one case, a foreign sensation in one case, headache/nausea in one case, and dissatisfaction in one case. In the cases in which adverse reactions occurred, the latanoprost-timolol maleate fixed-combination eyedrop regimen was discontinued and changed back to the latanoprost and timolol maleate eyedrop regimen. The adverse reactions subsequently disappeared quickly in all cases. The continuous activity rate for latanoprost-timolol maleate fixed-combination eyedrops is shown in Figure 2. The continuous activity rate of latanoprost-timolol maleate fixed-combination eyedrops was $72.8 \%$ at 12 months after administration, $65.4 \%$ at 24 months after administration, and $61.7 \%$ at 36 months after administration. Main reasons for discontinuation were appearance of adverse reactions, insufficient IOP decrease, and discontinuation of hospital visits. The periods corresponding to each of these reasons are shown in Figure 3. Discontinuation of hospital visits occurred throughout the 3 -year period. However, adverse reactions appeared in all of the cases within 9 months of administration, while $75.8 \%$ of the cases showing an insufficient IOP decrease were reported within 12 months of administration.

\section{Discussion}

In a previous report, the IOP-decreasing efficiency, visual field-preservation effect, and safety of changing a latanoprost

Table I Cases in which treatment was discontinued within 36 months after the change

Insufficient decrease in IOP

33 cases $(20.4 \%)$

Adverse reactions

II cases $(6.3 \%)$

Patient discontinued visits to the hospital

15 cases $(9.3 \%)$

Cataract surgery

3 cases $(1.9 \%)$

Abbreviation: IOP, intraocular pressure.

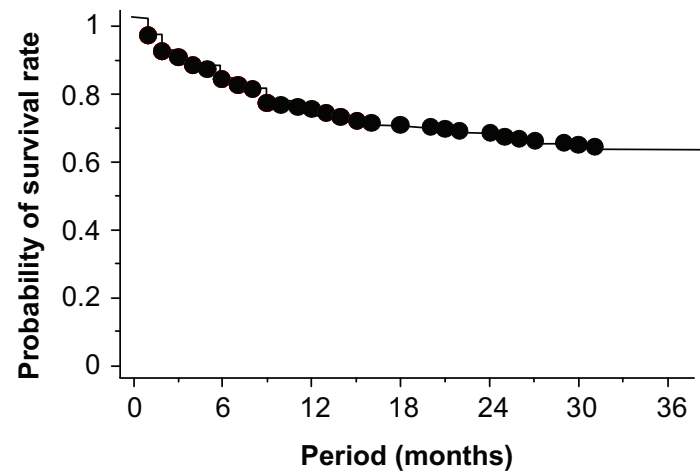

Figure 2 Continuous activity rate of latanoprost-timolol maleate fixed-combination eyedrops (Kaplan-Meier method).

and timolol maleate eyedrop regimen to the latanoprosttimolol maleate fixed-combination eyedrop regimen were investigated over a 1 -year period. ${ }^{2}$ No significant differences were found in the IOP before the change and at 3, 6, 9, and 12 months after the change. Dunker et al conducted a similar study, and reported no significant difference in the average IOP before the change $(18.4 \pm 3.4 \mathrm{mmHg})$ compared to 6 months after the change $(17.3 \pm 2.9 \mathrm{mmHg}) .^{3}$ Moreover, in a meta-analysis comparing the effectiveness of concomitantly used prostaglandin analog and timolol maleate eyedrops with that of prostaglandin analog-timolol maleate fixedcombination eyedrops, the fixed-combination eyedrops were less effective in reducing IOP than the concomitantly used eyedrops. ${ }^{8}$ The proportion of cases in which the eyedrops were changed because of insufficient IOP-decreasing efficacy increased by $20.4 \%$ in the present study when compared to that 1 year after the change $(12.3 \%){ }^{2}$ These data suggest that the IOP-decreasing effect of the $\beta$-blockers in fixedcombination eyedrops weakens after long-term administration of these drops.

Adverse reactions associated with latanoprost-timolol maleate fixed-combination eyedrops are hyperemia, a sensation of stimulation, foreign-body sensation, conjunctivitis, and

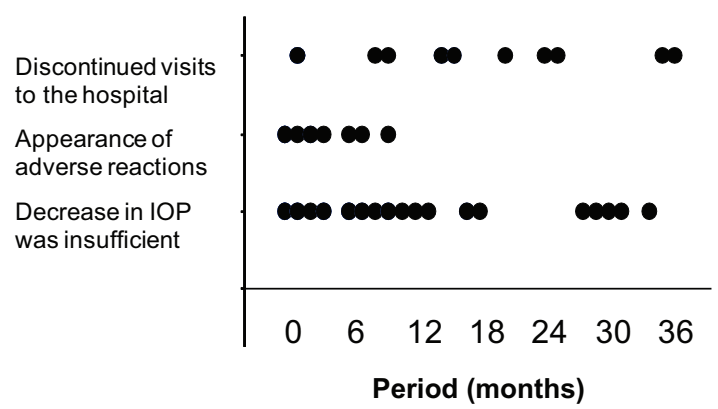

Figure 3 Reasons for discontinuation and discontinuation period. Each black circle represents one patient.

Abbreviation: IOP, intraocular pressure. 
corneal epithelial disorder. ${ }^{2-9}$ Numerous studies ${ }^{4-9}$ have reported a lack of serious adverse reactions, which is consistent with our findings in this study. Moreover, in the metaanalysis, the fixed-combination eyedrops were associated with a lower risk of hyperemia than the concomitantly used eyedrops. ${ }^{8}$ In the present study, it is thought that the appearance of adverse reactions decreased and their severity was lower when concomitantly used eyedrops were changed to fixed-combination eyedrops. Moreover, adverse reactions appeared in all of the cases within 9 months. Therefore, concerns over possible adverse effects may not be necessary if the patients do not show adverse reactions within 9 months.

We also previously investigated the preservation of the visual field by latanoprost-timolol maleate fixed-combination eyedrops. MD values before the change and at 12 months after the change were $-9.11 \pm 6.94 \mathrm{~dB}$ and $-10.08 \pm 7.24 \mathrm{~dB}$, respectively, and these values were not significantly different. ${ }^{2}$ Schwenn et al assessed patients with various types of glaucoma or ocular hypertension who had undergone a switch to a latanoprost-timolol fixed-combination eyedrop regimen from monotherapy, fixed-combination eyedrop therapy, or concomitant-use therapy; they used the mean defect value and Aulhorn stage classification to evaluate changes in the visual field defect for up to 24 months after the change. ${ }^{9}$ No significant difference was found between the mean defect value before the change and that obtained $6,12,18$, and 24 months after the change. Regarding the Aulhorn stage classification, the stage did not change in $59.0 \%$, deterioration occurred in $18.9 \%$, and improvement occurred in $22.1 \%$ of the patients at 24 months after the change. Because progression of the visual field defect is very slow, long-term follow-up is required. Most of the evidence showed maintenance in the visual field is focused on IOP reductions. ${ }^{10}$ However, IOP may show diurnal variations; therefore, evaluation of IOP is difficult. Peak IOP is related to progression of the visual field defect. ${ }^{11}$ Moreover, peak IOP is seen more often at daytime than at night. ${ }^{12}$ In this present study, IOP was measured only in the daytime. Therefore, IOP-decreasing efficacy and progression in the visual field was controlled and evaluated by IOP measured in the daytime.

In the present study, the observation period of the previous study ${ }^{2}$ was extended from 1 year to 36 months. The increase in the number of patients who discontinued the fixed-combination treatment regimen due to an insufficient IOP reduction could be attributed to the long-term $\beta$-blocker drift ${ }^{13}$ associated with treatment regimens involving latanoprost-timolol fixed-combination eyedrops.
A limitation of the present study is that there was no control group. The ideal design of this study would have been one in which patients who were using latanoprost and timolol maleate were concomitantly randomly blinded and divided into a latanoprost-timolol fixed-combination eyedrop group and a latanoprost and timolol maleate concomitantuse group. However, in the present study, we were not able to perform this procedure. The proportion of cases in which fixed-combination treatment was discontinued was rather high (38.3\%). Among these, treatment was discontinued in $20.4 \%$ of the cases due to an insufficient IOP reduction. The latanoprost-timolol maleate fixed-combination eyedrops could not be used for 3 years in any of the cases. These cases were excluded, and IOP was evaluated. Therefore, there was no change in IOP during 3-33 months, but it significantly decreased after 36 months.

In conclusion, when Japanese primary open-angle glaucoma and ocular hypertension patients switched from two agents (latanoprost and timolol maleate eyedrops) to latanoprost-timolol maleate fixed-combination eyedrops for 36 months, IOP was preserved and the defect in the visual field controlled. However, adverse reactions, such as ophthalmalgia, itching, or a sensation of stimulation, occurred or the IOP-decreasing effect was insufficient in approximately $27 \%$ of cases; therefore, careful follow-up is required after the change to fixed-combination eyedrops.

\section{Disclosure}

The authors report no conflicts of interest in this work.

\section{References}

1. Djafari F, Lesk MR, Harasymowycz PJ, Desjardins D, Lachaine J. Determinants of adherence to glaucoma medical therapy in a long-term patient population. J Glaucoma. 2009;18(3):238-243.

2. Inoue $\mathrm{K}$, Okayama R, Higa R, Wakakura M, Tomita G. Assessment of ocular hypotensive effect and safety 12 months after changing from an unfixed combination to a latanoprost $0.005 \%+$ timolol maleate $0.5 \%$ fixed combination. Clin Ophthalmol. 2012;6:607-612.

3. Dunker S, Schmucker A, Maier H. Tolerability, quality of life, and persistency of use in patients with glaucoma who are switched to the fixed combination of latanoprost and timolol. Adv Ther. 2007;24(2): 376-386.

4. Inoue K, Fujimoto T, Higa R, et al. Efficacy and safety of a switch to latanoprost $0.005 \%$ + timolol maleate $0.5 \%$ fixed combination eyedrops from latanoprost $0.005 \%$ monotherapy. Clin Ophthalmol. 2012;6: $771-775$.

5. Diestelhorst M, Larsson LI. A 12 week study comparing the fixed combination of latanoprost and timolol with the concomitant use of the individual components in patients with open angle glaucoma and ocular hypertension. Br J Ophthalmol. 2004;88(2):199-203.

6. Hamacher T, Schinzel M, Schölzel-Klatt A, et al. Short term efficacy and safety in glaucoma patients changed to the latanoprost $0.05 \% /$ timolol maleate $0.5 \%$ fixed combination from monotherapies and adjunctive therapies. Br J Ophthalmol. 2004;88(10):1295-1298. 
7. Polo V, Larrosa JM, Ferreras A, Borque E, Pablo LE, Honrubia FM. Effect on diurnal intraocular pressure of the fixed combination of latanoprost $0.005 \%$ and timolol $0.5 \%$ administered in the evening in glaucoma. Ann Ophthalmol (Skokie). 2008;40(3-4):157-162.

8. Quaranta L, Biagioli E, Riva I, et al. Prostaglandin analogs and timololfixed versus unfixed combinations or monotherapy for open-angle glaucoma: a systematic review and meta-analysis. J Ocul Pharmacol Ther. 2013;29(4):382-389.

9. Schwenn O, Heckmann B, Guzy C, Miller PJ. Long-term effect of latanoprost/timolol fixed combination in patients with glaucoma or ocular hypertension: a prospective, observational, noninterventional study. BMC Ophthalmol. 2010;10:21.

10. Collaborative Normal-Tension Glaucoma Study Group. Comparison of glaucomatous progression between untreated patients with normaltension glaucoma and patients with therapeutically reduced intraocular pressures. Am J Ophthalmol. 1998;126(4):487-497.
11. Konstas AGP, Quaranta L, Mikropoulos D, et al. Peak intraocular pressure and glaucomatous progression in primary open-angle glaucoma. J Ocul Pharmacol Ther. 2012;28(1):26-32.

12. Quaranta L, Konstas AG, Rossetti L, et al. Untreated 24-h intraocular pressures measured with Goldmann applanation tonometry vs nighttime supine pressures with Perkins applanation tonometry. Eye (Lond). 2012;24(7):1252-1258.

13. Boger WP 3rd, Puliafito CA, Steinert RF, Langston DP. Long-term experience with timolol ophthalmic solution in patients with open-angle glaucoma. Ophthalmology. 1978;85(3):259-267.
Clinical Ophthalmology

\section{Publish your work in this journal}

Clinical Ophthalmology is an international, peer-reviewed journal covering all subspecialties within ophthalmology. Key topics include: Optometry; Visual science; Pharmacology and drug therapy in eye diseases; Basic Sciences; Primary and Secondary eye care; Patien Safety and Quality of Care Improvements. This journal is indexed on

Submit your manuscript here: http://www.dovepress.com/clinical-ophthalmology-journal

\section{Dovepress}

PubMed Central and CAS, and is the official journal of The Society of Clinical Ophthalmology (SCO). The manuscript management system is completely online and includes a very quick and fair peer-review system, which is all easy to use. Visit http://www.dovepress.com/ testimonials.php to read real quotes from published authors. 